\title{
Adaptation of Rabbit Ventricular Cell Model to Reproduce Action Potentials in Isolated Papillary Muscle
}

\author{
Ask S Jensen, Cristian P Pennisi, Cristian Sevcencu, Jørn B Christensen, Jette E Kristiansen, \\ Johannes J Struijk \\ Aalborg University, Aalborg, Denmark
}

\begin{abstract}
Aims: We sought to establish a computational model of the rabbit ventricular action potential (AP) suitable for investigation of drug effects on the AP of the isolated rabbit papillary muscle.

Methods: Data consisted of transmembrane AP recordings from isolated right ventricular papillary muscles from 21 rabbits. An existing model of the rabbit ventricular $A P$ was adapted to reproduce experimental $A P$ amplitude and $A P$ duration $\left(A P D_{90}, A P D_{60}, A P D_{30}\right.$,) at multiple pacing rates by reduction of fast sodium current and overall depolarizing current. The resulting model was validated.

Results: At 2.0 and $0.5 \mathrm{~Hz}$ pacing respectively, the experimentally recorded $A P D_{90}$ was $113.9 \pm 11.2 \mathrm{~ms}$ and $150.2 \pm 13.1 \mathrm{~ms}$. The adapted model produced an $A P D_{90}$ of $130.5 \mathrm{~ms}$ and $142.5 \mathrm{~ms}$. Validation of the adapted model showed that, while there was a substantial adaptation of the model APD to experimental data, model stability was maintained, and internal Ca dynamics and responses to stimuli were not substantially affected.

Conclusion: A model of the rabbit ventricular AP was adapted in order to reproduce experimental $A P$ recordings from the isolated right ventricular rabbit papillary muscle. This model is useful for analysis of drug effects on the rabbit papillary AP.
\end{abstract}

\section{Introduction}

The isolated papillary muscle is a standard preparation for investigation of drug induced effects on the ventricular AP. To facilitate model based analysis of mechanisms underlying drug effects in this preparation, we adapted the rabbit ventricular AP model published by Shannon et al. [1] to reproduce parameters of our baseline transmembrane AP recordings. The adapted model will be applied in future investigation of drug effects.

\section{Methods and materials}

Data collection was previously described in detail [2]. APs were recorded in right ventricular papillary muscles isolated from 21 female New Zealand white rabbits. For all rabbits, APs were recorded at baseline without any drugs at pacing rates of 0.5 and $2.0 \mathrm{~Hz}$. Sequences of baseline recordings were obtained from approximately eight different cells from each rabbit, and a median AP was calculated for each cell. We used a current MatLab implementation of the Shannon rabbit ventricular AP model published online. Adaptation of the model was done by changes in the maximal current conductance of membrane currents. The major transmembrane currents investigated were: the fast inward sodium current $\left(\mathrm{I}_{\mathrm{Na}}\right)$, the rapid and slow components of the delayed rectifier potassium current $\left(\mathrm{I}_{\mathrm{Kr}}, \mathrm{I}_{\mathrm{Ks}}\right)$, the fast and slow components of the transient outward potassium current $\left(\mathrm{I}_{\mathrm{tof}}, \mathrm{I}_{\mathrm{tos}}\right)$, the inward rectifier potassium current $\left(\mathrm{I}_{\mathrm{K} 1}\right)$, the L-type calcium current ( $\left.\mathrm{I}_{\mathrm{CaL}}\right)$, the sodium-potassium pump $\left(\mathrm{I}_{\mathrm{NaK}}\right)$, and the sodium-calcium exchanger $\left(\mathrm{I}_{\mathrm{NaCa}}\right)$.

\subsection{Baseline adaptation}

The default model AP displayed longer $\mathrm{APD}_{90}$ and greater amplitude compared to experiment. The mean and $95 \%$ confidence interval of measured AP amplitude (defined as the maximal value of the upstroke) was $21.4 \pm$ $2.5 \mathrm{mV}$ and $23.8 \pm 2.4 \mathrm{mV}$ at 2.0 and $0.5 \mathrm{~Hz}$ pacing respectively. The default model AP amplitude was 42.7 $\mathrm{mV}$ and $43.7 \mathrm{mV}$. Before adaptation to APD, the model AP amplitude was reduced by reduction in $\mathrm{I}_{\mathrm{Na}}$ current conductance determined by minimization of the error between model and measured AP amplitude.

The mean and $95 \%$ confidence interval of measured $\mathrm{APD}_{90}$ was $113.9 \pm 11.2 \mathrm{~ms}$ and $150.2 \pm 13.1 \mathrm{~ms}$ at 2.0 and $0.5 \mathrm{~Hz}$ pacing respectively. The model $\mathrm{APD}_{90}$ was $190 \mathrm{~ms}$ and $221.5 \mathrm{~ms}$ and was adapted to experiment by reduction of the $\mathrm{I}_{\mathrm{CaL}}, \mathrm{I}_{\mathrm{NaK}}$, and $\mathrm{I}_{\mathrm{NaCa}}$ currents by multiplication of the current conductances with an identical factor to minimize the error between model and measured $\mathrm{APD}_{90}$ across both frequencies. This approach was based in part on the results of the sensitivity analysis as described in the discussion. 

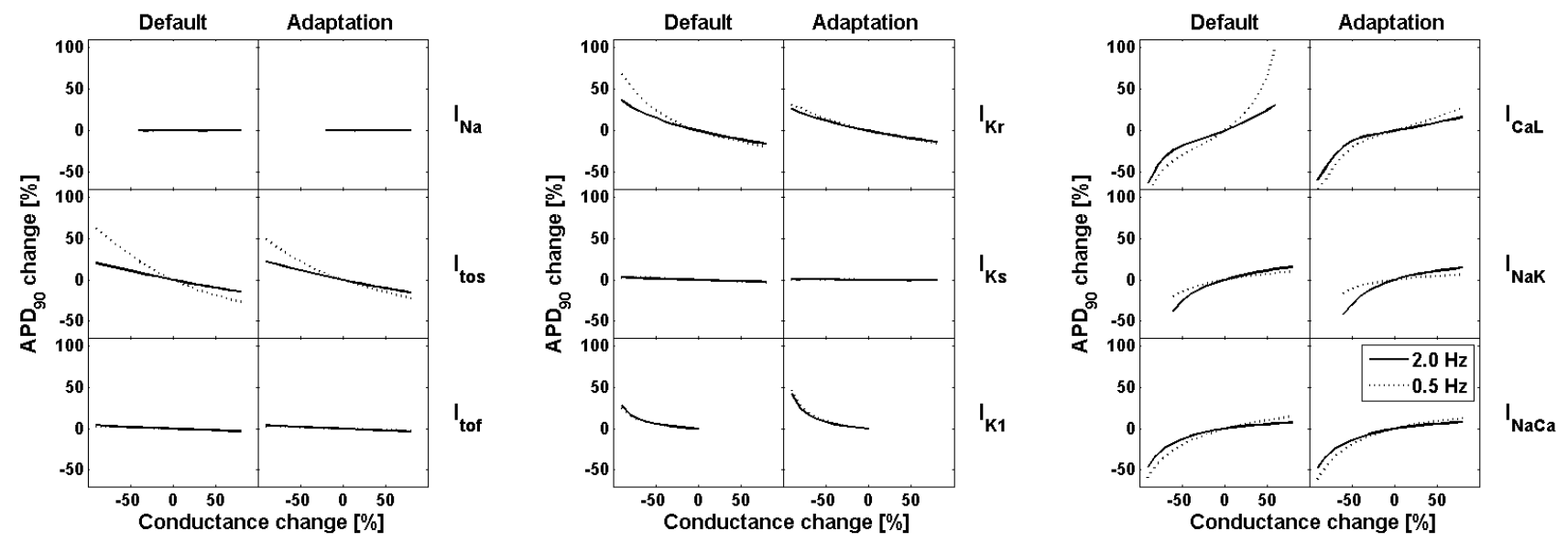

Figure 1: sensitivity analysis of $\mathrm{APD}_{90}$ change relative to baseline caused by reduction or increase in the conductance of major ion currents. Left subcolumns show the sensitivity analysis for default parameters, while the right subcolumns show results after adaptation of the model to experimental baseline measurements. Solid lines indicate changes at $2.0 \mathrm{~Hz}$ pacing while dotted lines indicate changes at $0.5 \mathrm{~Hz}$ pacing. Values are expressed as a percentage of baseline APD90.
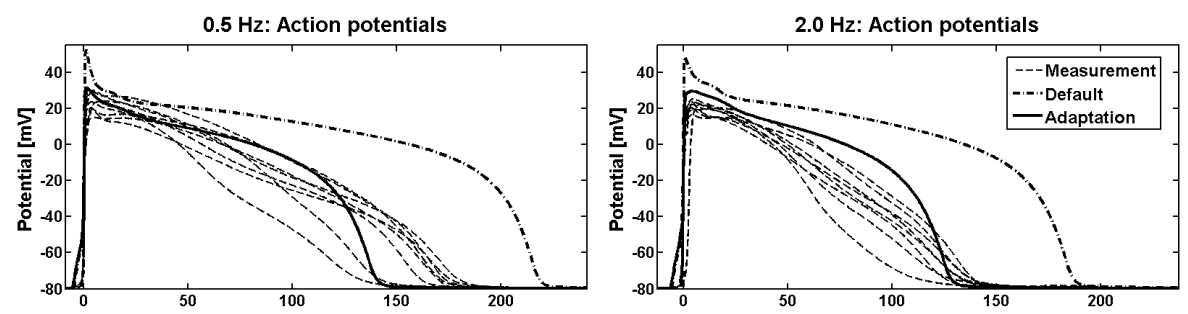

Figure 2: steady state model AP before and after baseline adaptation at 0.5 and $2.0 \mathrm{~Hz}$. Also shown are example AP recordings from eight different rabbits.

The adapted model was validated using tests similar to those used to validate the original model as reported by Shannon et al. [1]. This was done in comparison with the default model and literature data. The frequency dependence of APD ${ }_{90}$, cytosolic Na concentration $\left(\mathrm{Na}_{\mathrm{i}}\right)$, and total $\mathrm{Ca}$ concentration in the sarcoplasmic reticulum (CasRT, SR) was investigated. We also investigated the time-course of SR Ca release and uptake, and the balance between mechanisms competing to remove $\mathrm{Ca}$ from the cytoplasm following Ca release. These mechanisms were: $\mathrm{I}_{\mathrm{NaCa}}$, the SR Ca pump ( $\mathrm{I}_{\mathrm{CaSR}}$ ), and the sarcolemmal Ca pump ( $\mathrm{I}_{\mathrm{CasL}}$ ). In order to test the stability of the model we also investigated the response in CasRT and cytosolic $\mathrm{Ca}$ $\left(\mathrm{Ca}_{\mathrm{i}}\right)$ transients to either a bolus of $0.4 \mathrm{mM}$ free $\mathrm{Ca}$ added to the SR or an instantaneous removal of $0.4 \mathrm{mM}$ free $\mathrm{Ca}$ from the SR. The change in APD induced by change in pacing rate was compared with our measurements.

\section{Results}

The adaptation of the model was evaluated. A minimal difference between model and measured AP amplitude was obtained by a $50 \%$ reduction in $\mathrm{I}_{\mathrm{Na}}$ conductance. This fit to baseline signals reduced the amplitude of the model AP from 42.7 to $23.8 \mathrm{mV}$ at $2.0 \mathrm{~Hz}$ pacing and from 47.3 to $26.0 \mathrm{mV}$ at $0.5 \mathrm{~Hz}$ pacing. In both cases this was within the $95 \%$ confidence interval, and no change in model $\mathrm{APD}_{90}$ occurred, see Figure 1.

The model was adapted to baseline $\mathrm{APD}_{90}$ by a reduction in $\mathrm{I}_{\mathrm{CaL}}, \mathrm{I}_{\mathrm{NaK}}$, and $\mathrm{I}_{\mathrm{NaCa}}$ conductance. The minimal difference between model and measured $\mathrm{APD}_{90}$ was achieved by $40 \%$ reduction in these conductances. This adaptation reduced $\mathrm{APD}_{90}$ from 190.0 to $130.5 \mathrm{~ms}$ at $2.0 \mathrm{~Hz}$ pacing and from 221.5 to $142.5 \mathrm{~ms}$ at $0.5 \mathrm{~Hz}$ pacing. The model baseline $\mathrm{APD}_{90}$ resulting from this adaptation was within the $95 \%$ confidence interval of experimental measurement at $0.5 \mathrm{~Hz}$ pacing, but at $2.0 \mathrm{~Hz}$ pacing the modeled $\mathrm{APD}_{90}$ was slightly longer. The effect of the adaptation on the APD is shown in Figure 2.

\subsection{Validation}

The results of the validation are shown in Figure 3. The time-course and magnitude of Ca release from the SR was not strongly affected. The fractional release of Ca as a percentage of total Ca content changed from 27 to $24 \%$ at $0.5 \mathrm{~Hz}$ pacing. The balance between mechanisms competing to remove $\mathrm{Ca}$ from the cytoplasm was also 

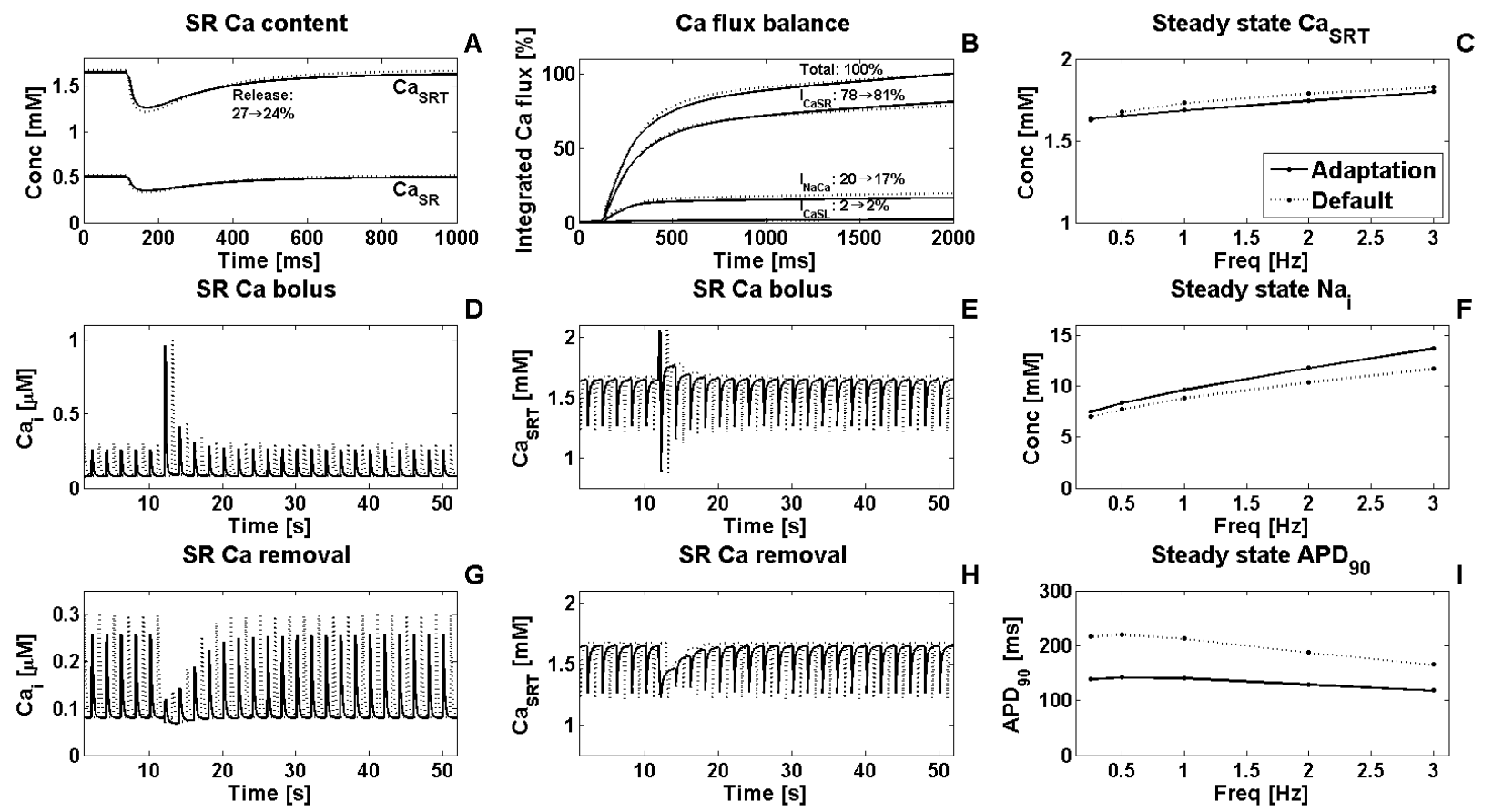

Figure 3: Results of validation. A: Ca release from SR following excitation. B: Ca removal fluxes. C: pacing rate dependence of total SR Ca in steady state. D: cytosolic Ca transient response to Ca bolus. E: total SR Ca response to Ca bolus. F: pacing rate dependence of cytosolic Na in steady state. G: cytosolic Ca transient response to Ca removal. H: total SR Ca response to Ca removal. I: pacing rate dependence of $\mathrm{APD}_{90}$ in steady state. In parts $\mathrm{D}, \mathrm{E}, \mathrm{G}$, and $\mathrm{H}$ the results of the default model are shifted by $1 \mathrm{~s}$ to improve legibility.

almost unchanged at $0.5 \mathrm{~Hz}$ pacing. The fraction of total Ca removal attributable to the SR pump uptake changed from 78 to $81 \%$. The Ca removal attributable to $\mathrm{I}_{\mathrm{NaCa}}$ changed from 20 to $17 \%$. The steady state rate dependence of total SR Ca content was highly similar between models. The steady state level of cytosolic $\mathrm{Na}$ was increased by the adaptation: from 10.33 to $11.65 \mathrm{mM}$ (12.7 \%) at $2 \mathrm{~Hz}$ pacing and from 7.79 to $8.41 \mathrm{mM}(8.0$ $\%)$ at $0.5 \mathrm{~Hz}$. The APD 90 was shortened as intended, and the steady state rate dependence of $\mathrm{APD}_{90}$ became slightly less steep. The amplitude of cytosolic $\mathrm{Ca}$ following excitation at $0.5 \mathrm{~Hz}$ pacing decreased from 0.30 to $0.26 \mu \mathrm{M}(-13.6 \%)$. After instantaneous addition or removal of free $\mathrm{Ca}$ in the SR, cytosolic Ca transients and SR Ca levels quickly returned to steady state over a time course identical to that for the default model.

The effects of the adaptation were also evaluated through sensitivity analysis, see Figure 1 . The adaptation did not substantially change the sensitivity of $\mathrm{APD}_{90}$ towards change in the $\mathrm{I}_{\mathrm{Na}}$, $\mathrm{I}_{\mathrm{tof}}$, and $\mathrm{I}_{\mathrm{Ks}}$ currents. The prolongation caused by high degrees of $\mathrm{I}_{\mathrm{K} 1}$ inhibition increased. The impact of $\mathrm{I}_{\mathrm{tos}}$ and $\mathrm{I}_{\mathrm{Kr}}$ at $2 \mathrm{~Hz}$ pacing did not change greatly, but the reverse rate dependence of prolongation due to $\mathrm{I}_{\text {tos }}$ inhibition was reduced moderately, and the reverse rate dependent prolongation due to $\mathrm{I}_{\mathrm{Kr}}$ inhibition was almost completely abolished. The sensitivity to moderate $\mathrm{I}_{\mathrm{CaL}}$ changes was reduced slightly, and the reverse rate dependent prolongation due to great increases in $\mathrm{I}_{\mathrm{CaL}}$ was dramatically reduced. The direct rate dependence of changes due to $\mathrm{I}_{\mathrm{NaK}}$ was slightly increased, while the impact of $\mathrm{I}_{\mathrm{NaCa}}$ changed little.

\section{Discussion}

Experimentally recorded APs were found to have baseline durations shorter than the default model. However, reported values of baseline rabbit APD span a relatively large range, as the APD depends on factors including age, gender, tissue type, anatomical region, and experimental methods and conditions. Romero et al. reports examples of baseline $\mathrm{APD}_{90}$ values ranging between 123 and $190 \mathrm{~ms}$ [3]. It was sought to adapt the model to baseline APD through minimal changes of the transmembrane currents, resulting in APs with a duration and rate dependence agreeing with experiment and the literature. In addition it was sought to maintain model stability and to avoid introducing unexpected behaviors. As the APD depends on the balance between repolarizing and depolarizing currents, reduction in model APD could be attained though either increasing the repolarizing current or decreasing the depolarizing current. These currents were selected based on the outcome of the sensitivity analysis. This analysis indicated that the repolarizing currents $\mathrm{I}_{\mathrm{tof}}$ and $\mathrm{I}_{\mathrm{Ks}}$ had only negligible 
impact on APD, see Figure 1. The $\mathrm{I}_{\mathrm{K} 1}$ current had some impact on APD, but increased transient $\mathrm{I}_{\mathrm{K} 1}$ current during initial depolarization abolished AP triggering. The main repolarizing currents, $\mathrm{I}_{\mathrm{tos}}$ and $\mathrm{I}_{\mathrm{Kr}}$, both displayed a substantial degree of rate dependent APD prolongation when inhibited. However, APD $_{90}$ response to increases in these currents was relatively modest, such that substantial increase would be required to impact the adaptation to baseline. The $\mathrm{I}_{\mathrm{Kr}}$ current was not increased, as the model $\mathrm{APD}_{90}$ prolongation due to $\mathrm{I}_{\mathrm{Kr}}$ inhibition was very similar to that recorded experimentally for a pure $\mathrm{I}_{\mathrm{Kr}}$ blocker [4]. The $\mathrm{I}_{\text {tos }}$ current was also determined to be unsuitable due to the strongly reverse rate dependent impact on APD90. Increase in $\mathrm{I}_{\text {tos }}$ would decrease the rate dependence of APD at baseline contrary to measurements, and the rate dependence of prolongation due to inhibition would become unrealistically great. Consequently, an increase in repolarizing current was evaluated to be unsuitable for APD adaptation, and instead the adaptation was carried out by reduction in overall depolarizing current by reduction of $\mathrm{I}_{\mathrm{CaL}}, \mathrm{I}_{\mathrm{NaK}}$, and $\mathrm{I}_{\mathrm{NaCa}}$ conductances.

The validation of the model indicated that, despite the relatively substantial change in $\mathrm{APD}_{90}$, the inner mechanisms of the model behave very similarly to the default model. The release of Ca from the SR was only affected slightly by the adaptation and agrees well in time course and magnitude with experimental data [5], similarly to the default model [1]. With regards to $\mathrm{Ca}$ transport, the balance between Ca removal fluxes is one of the most important aspects and critical to the models predictive power, as the balance in steady state essentially determines the gain of the excitation-contraction coupling (the amount of $\mathrm{Ca}$ released from SR relative to the amount introduced by $\mathrm{I}_{\mathrm{CaL}}$ ) [1]. The validation showed that despite changes to $\mathrm{I}_{\mathrm{CaL}}$ and $\mathrm{I}_{\mathrm{NaCa}}$, the balance changed very little. In addition, it is also in very good agreement with experimental data, similarly to the default model [1]. The adaptation also induced changes in the SR Ca load and cytosolic Na concentration. However, relative to the magnitude of APD changes, these changes in ionic concentration were small. Model stability was maintained, as the adapted model reliably arrived at a history-independent steady state under physiologically relevant conditions. Perturbation of the steady state by sudden addition or subtraction of $\mathrm{Ca}$ in the SR resulted in a rapid return to steady state identical to the default model. These results indicate that the feedback loops which ensure the stability of the real myocardial cell and which are described by the Shannon model are also maintained in the adapted model.

The changes induced by adaptation of the model to baseline recordings were investigated. The $\mathrm{APD}_{90}$ of the adapted model was similar to measurement, although the response of model $\mathrm{APD}_{90}$ to rate change was somewhat smaller than in experiment. The model AP shape had more pronounced plateau with a rapid final repolarization compared to AP recordings. However, a less pronounced plateau is commonly found in the literature for recordings of rabbit APs in isolated multicellular preparations, examples include [6]. The sensitivity analysis indicated that sensitivity of $\mathrm{APD}_{90}$ to the conductances of transmembrane currents was similar between the default and adapted models. Thus, the adaptation did not drastically alter the impact of current inhibitions, and no new unexpected behaviors were identified. The greatest difference was that the model became less vulnerable towards excessive reverse rate-dependent prolongation as observed for an increase in $\mathrm{I}_{\mathrm{CaL}}$ current in the default model. The adaptation also reduced the reverse rate dependency of APD prolongation due to $\mathrm{I}_{\mathrm{Kr}}$ inhibition. This may have been related to the short baseline APD.

\section{Conclusion}

The Shannon model of the rabbit ventricular AP was adapted to measured baseline AP features. The adapted model produces APs with duration similar to experiments while also maintaining the stability and key behaviors of the original model. The behavior of its internal mechanisms are in good agreement with experimental data. The adapted model serves as a tool to analyze experimentally measured drug effects.

\section{References}

[1] Shannon TR, Wang F. A mathematical treatment of integrated ca dynamics within the ventricular myocyte. Pharm Res 2004;87(5):3351-71.

[2] Jensen AS, Struijk JJ. Differential effects of thioridazine enantiomers on action potential duration in rabbit papillary muscle. Eur J Pharmacol 2015;747:7-12.

[3] Romero L, Carbonell B. Systematic characterization of the ionic basis of rabbit cellular electrophysiology using two ventricular models. Prog Biophys Mol Biol 2011; 107(1):60-73.

[4] Lengyel C, Iost N. Pharmacological block of the slow component of the outward delayed rectifier current (IKs) fails to lengthen rabbit ventricular muscle QTc and action potential duration. Br J Pharmacol 2001;132(1):101-10.

[5] Shannon TR, Guo T. Ca2+ scraps: local depletions of free $\mathrm{Ca} 2+$ in cardiac sarcoplasmic reticulum during contractions leave substantial Ca2+ reserve. Circ Res 2003;93(1):40-5.

[6] Biagetti MO, Quinteiro RA. Gender differences in electrical remodeling and susceptibility to ventricular arrhythmias in rabbits with left ventricular hypertrophy. Heart Rhythm 2006;3(7):832-9.

Address for correspondence.

Johannes Jan Struijk.

Fredrik Bajers Vej 7, 9220 Aalborg Ø, DK. jjs@hst.aau.dk 